\title{
A Methodology for Quality Control Evaluation for Laminated Composites Manufacturing
}

Universidade Federal de Minas Gerais Mechanical Engenineering Av. Antônio Carlos, 6627

31270-901 Belo Horizonte, Minas Gerais. Brazil aavila@netuno.lcc.ufmg.br
Composites made by hand lay-up are directed influenced by some fabrication factors, e.g. the stacking sequence, fiber volume fraction, as well as the cure process This study takes into consideration an E-glass/epoxy plain weave woven fabric composite made by hand lay-up. After the stacking sequence is completed, three sets of plates are selected and each one is cured differently. The three selected cure processes are: cure on air, cure vacuum assisted, and cure under compression. A variance study based on stiffness from ASTM D $3039 / 3039 M$ tensile tests is performed to check the statistical differences caused by the cure processes. Additionally, a microscopic analysis is performed to identify the voids formation rate. The coupling between macro and micro-mechanical analysis is done by a non-dimensional coefficient which is able to capture the rate of defects generated by each cure process.

Keywords: Composite materials, micro-mechanics, macro-mechanics, manufacturing quality control evaluation

\section{Introduction}

According to Gutowski (1997), the most important manufacturing process of composites applied to aerospace industry is the hand lay-up of prepregs and autoclave cure. Although the manufacturing processes have been undergoing constant technical changes, the hand lay-up still persists as the method in use for more than half of all advanced aerospace composite structures. Its large use results from the extreme flexibility which allows the manufacturing of a large variety of shapes. Additionally, hand layup does not require large capital investments. In this type of manufacturing two distinct steps can be established; the first one consists of fibers impregnation and stacking, and the second one is the cure procedure.

During the last two decades most researchers focused on the composite's mechanical properties estimation. The manufacturing process was somehow neglected to a secondary place. Moreover, the cure process was treated for a long time as if it was a mere recipe, but this behavior is changing. To overcome this "cultural barrier", two approaches are commonly used. The first one focuses on experimental data, e.g. Um et al (2002), where the cure kinematics was studied by differential scanning calorimetry (DSC). However, this technique is not suitable for low temperature cure where the reaction rate is too slow. As for the second one, numerical simulations have been developed with good results, e.g. Mantell and Springer (1992), Blest et al (1999). A more comprehensive analysis was done by Fernlund et al. (2002) where, in addition to the experiments, a numerical analysis was performed. In all these cases the main focus was on the manufacturing process.

Nonetheless, to improve the composite overall performance, it is needed not only to consider the mechanical properties estimation models but also the manufacturing processes with their advantages and limitations. Daniel and Abot (2000) linked the two areas by taking into account the problem of void formation on laminated composites. According to them, voids can be formed either by mechanical entrapment of air or by nucleation from vapors or gases. The mechanical entrapment could be due to entrained gas bubbles from resin mixing operations, bridging from large particles, voids from wandering tows, broken fibers, to air pockets and wrinkles created during the lay-up. As acclaimed by Mallick (1988), void formation causes stiffness and strength reduction. He went further

Paper accepted June, 2005. Technical Editor: Atila P. Silva Freire. trying to correlate void formation with two key factors, i.e. time and temperature during the resin cure. One of his conclusions was that temperature and time were inversely proportional to each other. According to Yoshida et al (1986), the presence of voids leads to a severe degradation on strength and stiffness. In some composite manufacturing processes, e.g. resin transfer molding (RTM), resin film infusion (RFI), and hand lay-up, the problem of void formation is also related to the manufacturing process parameters such as fiber volume fraction, fiber permeability, and resin viscosity. Han et al (2003) performed an experimental study on RFI manufacturing of stitched stiffened composite plates, and their conclusion was that void formation is mostly related to the two-dimensional resin flow inside the mould. Moreover, the stitches location can also induce void formation, as they somehow block the resin flow. Kang et al. (2000) had shown that void formation in RTM is directly related to imperfections on fiber architecture which leads to a non-uniform permeability. As a consequence of this non-homogeneity, the resin flow is perturbed bringing as a consequence void formation. For the hand lay-up procedure, however, the fiber impregnation is related to resin flow and fiber permeability. In all cases, RFI, RTM and hand lay-up, void formation can be induced in certain directions due to the manufacturing processes. For instance, a bad selection of stitches locations can lead to a narrow resin path that can trim down the resin flow which can direct to a series of voids in a preferential direction.

Taking into consideration all the points discussed above, it is possible to conclude that there is a need for a study on manufacturing processes effects on mechanical properties. The purpose of this paper is to make a statistical study on hand lay-up manufacturing process with cure to air, under compression and assisted by vacuum and their influence on composite's stiffness and strength. Additionally, a new methodology to estimate the rate of defects generated during each manufacturing process is proposed

\section{The Hand Lay-Up Performance Analysis}

Gutowski (1997) stated that it is virtually impossible to obtain a defect-free composite. Therefore, a good parameter for comparison between manufacturing processes is the level of defects generated during each manufacturing process. The composite designer must have in mind that variations on stiffness and strength can be due to the choice of the manufacturing process. According to Kassapoglou (1999), the use of the same technology with slight differences can lead into completely dissimilar mechanical properties. To prove his point, Kassapoglou mentioned the composite wing box case. This 
wing box was made by hand lay-up and parts of it were submitted to different co-curing cycles. As a consequence, complete dissimilar mechanical properties from one section to another were obtained Based on these arguments, it is possible to concluded that there is a need for a performance study taking into consideration the manufacturing parameters, in special the cure procedures.

One model for composite manufacturing evaluation was introduced by Avila et al (2001), who proposed a non-dimensional coefficient, called Yoke Ratio, where a relation between the actual and the designed stiffness was established. Later on, Morais and Avila (2003) extended the concept and the Generalized Yoke Ratio (GYR) was developed. In their original work, the GYR mathematical representation is given by:

$$
\eta=\Omega\left\|\lambda_{x} \hat{i}, \lambda_{y} \hat{j}, \lambda_{z} \hat{k}\right\|
$$

and

$$
\lambda_{s}=\frac{\partial \kappa\left(\mathrm{E}_{\mathrm{s}}^{\text {Macro }}\right)}{\partial \mathrm{s}} \frac{\partial \mathrm{s}}{\partial \kappa\left(\mathrm{E}_{\mathrm{s}}^{\text {Micro }}\right)} \quad s=x, y, z
$$

where $\kappa\left(\mathrm{E}_{\mathrm{x}}{ }^{\text {macro }}\right)$ is the vector which contains the Young's modulus variation, evaluated at macroscopic level, at $\mathrm{x}$ direction. Notice $\wedge \wedge \wedge$

$i, j, k$ that are the three mutually orthogonal unit vectors associated to the $\mathrm{x}, \mathrm{y}$, and $\mathrm{z}$ directions, and $\Omega$ is the degrees-of-freedom nondimensional coefficient. The superscript micro and macro are indication of micro and macro-mechanics analysis. Moreover, the symbol $\|$ || denotes the L-2 norm, also known as the Frobenius norm (Golub and Van Loan, 1996), defined as:

$$
\|A\|=\left[\sum_{i=1}^{m} \sum_{j=1}^{n}\left|a_{i j}\right|^{2}\right]^{1 / 2}
$$

As the GYR definition involves two scales of analysis, macro- and micro-mechanical, the proposed methodology must reflect both. The macro-mechanical analysis is based on the tensile test described by the ASTM D 3039/3039M-00 (2000) standard, while the micro-mechanical analysis involves not only the porosity determination via optical microscopy but also a mathematical modeling based on unit cell approach. The linkage between micro- and macro-mechanics is performed considering the following method. The first step is the ASTM D 3039/3039M-00 specimen preparation and testing. From the results, a vector $\kappa$ is created. This vector contains the stiffness variation based on tensile tests. From each ASTM specimen group a set of longitudinal and transversal cuts are performed and the composite porosity and packing factor is obtained via optical microscopy. Once these data are obtained, a micro-mechanical analysis is performed using the mmTEXlam code (Challa and Shivikumar, 2001). From the numerical simulation, a similar $\kappa$ vector is constructed based on micro-mechanics. Based on these two vectors, the GYR can be calculated.

The interesting feature about the GYR is its capacity of representing the rate of defects or voids generated during the manufacturing process. Notice that the micro-mechanical model is considered as a defect-free model, which will lead to stiffness upper bound value. On the other hand, the results given by the GYR can only be interpreted in an average sense as the Frobenius norm acts as a mathematical function of homogenization. To be able to capture how the mechanical properties sensibility to void formations a different concept must be introduced.

Before introducing the new parameter, some issues must be addressed. In some composites manufacturing processes the void formation is directly influenced by fabrication parameters. According to Patel et al. (1995), RTM and RFI are some of these manufacturing processes in which parameters such as fiber permeability, resin viscosity, resin velocity have influence on void fraction. They also stated that void sizes in the resin flow direction decrease with the increase of flow rate. For the hand lay-up case, however, a non-uniform pressure during the manual resin impregnation can lead to a path of fiber imperfections that brings non-homogeneous fiber permeability and, as consequence, void formation. Therefore, it seems reasonable to consider void formation as a directional quantity. To be able to numerically define this quantity, the three mutual orthogonal axes $(x, y, z)$ are coincident to the composite three principal axes $\mathrm{X}_{1}, \mathrm{X}_{2}, \mathrm{X}_{3}$. Moreover, the composite is considered as an orthotropic material. As a consequence, the directional yoke ratio (DYR) can now be defined in its vector form:

$$
\eta_{i}=\frac{\partial \kappa\left(\mathrm{E}_{\mathrm{i}}^{\text {Macro }}\right)}{\partial \mathrm{x}_{\mathrm{i}}} \frac{\partial \mathrm{x}_{\mathrm{i}}}{\partial \kappa\left(\mathrm{E}_{\mathrm{i}}^{\text {Micro }}\right)} i=1,2,3
$$

As the composite is orthotropic, a susceptibility coefficient $(\xi)$ can be associated to each of the three principal directions. By assuming this condition, we can track the stiffness variation as a function of void formation in each one of the three principal directions. The $\xi$ mathematical representation is defined as:

$$
\xi_{i}=\left(\frac{\partial \eta_{i}}{\partial v_{i}}\right) \quad i=1,2,3
$$

where $v$ is the amount of voids intercepted by each principal axis. This parameter will lead to information about the manufacturing process homogeneity, i.e., any problem during the manufacturing that could lead to void formation can be detected by the number of voids generated in each direction. The coefficient of sensibility represents the DYR variation as a function of the percentage of voids in each direction, or simply the slope of the DYR versus voids percentage plot. $\xi$ represents the composite sensibility, in terms of stiffness variation and void formation, to a specific manufacturing process.

Although the non-dimensional coefficients can provide an estimation of the manufacturing process effects on composite's mechanical properties, to be able to carry out the hand lay-up performance analysis a study on statistical consistency must be done. The design of experiments methodology proposed by Montgomery (2001) is applied to find the number of samples needed for a statistically significant data. First, a pilot sample of 10 specimens for each manufacturing process is prepared. Then, the tensile tests are performed and the stiffness and strength are obtained. For the Young's modulus and the ultimate stress, the mean value $(\mu)$, standard deviation $(\sigma)$ and the maximum error (E') are computed.

The procedure suggested by Montgomery (2001) takes into consideration the sample size of various groups of specimens, with different mean values. In this case, we have:

$$
\Phi^{2}=\frac{n \cdot \sum_{i=1}^{k} \pi^{2}}{\Delta \cdot \sigma^{2}}
$$


where

$$
\tau=\overline{x_{i}}-\bar{x}
$$

$\Delta$ is the number of processes compared, and $\overline{x_{i}, \bar{x}}$ are the mean value for each sample and for all populations together, respectively, while, the estimate variance is defined by $\sigma^{2}$. Once the $\Phi$ parameter is obtained and the degrees-of-freedom are known, it is possible to fit the sample size ( $n$ ) using the operating characteristic curves (OC curves).

The largest value of $n$ is assumed as the uniform sample size Tensile tests are performed and the following parameters are evaluated: Young's modulus and ultimate strength. To be able to carry out a comparison among the different manufacturing parameters, a variance analysis was applied. Montgomery (2001) mentioned that for a normal distribution, the ANOVA model is more convenient while when a normal distribution is not applicable, a non-parametric model, e.g. Kruskal-Wallis, is more adequate. These two methodologies are used in this study.

\section{Experimental Procedures}

The performance study is carried out considering three different cure processes of a hand lay-up composite, i.e. cure on air, cure under compression and cure vacuum assisted. The composite is made of 8 layers of plain weave woven fabric E-glass (Texiglass, 2002), surrounded by an epoxy matrix (Huntsman, 2005) with a nominal weight fraction of fibers of $50 \%$. During the compression procedure, a pressure equivalent to one atmosphere is applied, while for the vacuum bagging operations a pressure close to $30 \mathrm{mmHg}$ is used. For both cases, the pressure was kept constant up to 24 hours. Notice that according to the resin manufacturer, Hunstman (2005), after 24 hours at room temperature the resin is completely cured.

The tensile tests are performed considering the ASTM D 3039/3039M-00 standard. The methodology applied to compute the elastic moduli via micro-mechanical models, however, involves not only the recognition of a unit cell but also the use of optical microscopy techniques to measure the fiber volume fraction, the distances between the tow fibers, the packing factor, and the filaments diameter. The micro-mechanical model used is based on the unit cell approach for woven fabric composites as defined in Challa and Shivakumar (2001). The public domain micromechanical code mmTEXlam is employed to compute the elastic moduli estimations. To measure the unit parameter, a series of longitudinal and transversal cuts are performed for each group. The optical microscopy samples have an area equivalent to $100 \mathrm{~mm}^{2}$. The procedure of encapsulating, polishing and attacking the surface followed the one proposed by Sawyer and Grubb (1996). The image measure software QUANTIMET $^{\circledR}$ is used to obtain the measurements. At least 10 measurements in each specimen are performed, and the average values are computed.

\section{Data Analysis}

Although the ultimate strength values are evaluated during the tensile tests, we will focus on stiffness as the relationship between micro- and macro-mechanical analysis is performed in terms of stiffness. The data analysis must be divided in three categories, i.e. micro-mechanical modeling and optical observations, macromechanical results and statistical analysis, and finally the nondimensional parameters calculations.

The next step is the design of experiment. From each laminated plate, five ASTM D 3039/3039 M specimens are prepared. On the experiment first phase, sample size estimation, 10 specimens for each type of cure are made and tested. Following Montgomery (2001) the probability of type I error, often called the level of significance of the test, $(\alpha)$ and the probability of type II error $(\beta)$ are fixed equal to 0.01 and 0.05 , respectively. The sample size calculation is performed considering separately Young's modulus on axial direction (stiffness) and ultimate stress (strength). By using the operating characteristic curves from Montgomery, it is possible to compute a sample size population $(n)$ equals to 23 with a probability $(1-\beta)$ of $95 \%$. To be on a safe side, a sample size of 25 specimens is selected.

As it can be observed in Figures 1A-1C, the typical fracture shape does not change much with the cure process. However, a larger delamination on the gage is noticed on those specimens from cure under vacuum.

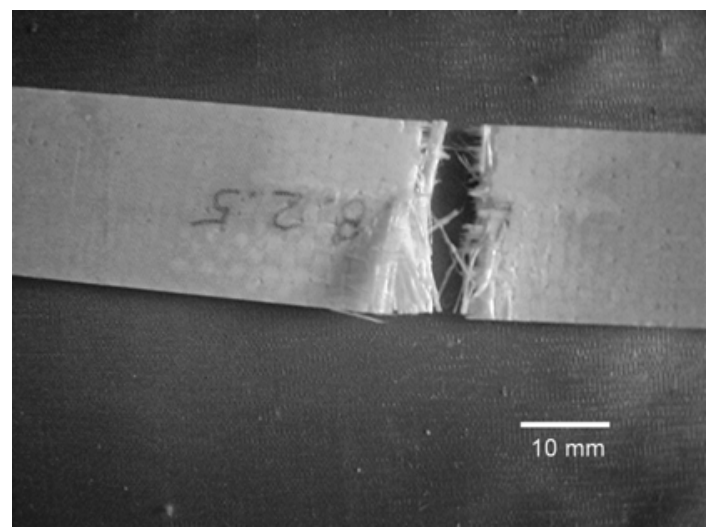

(a) Cure on air

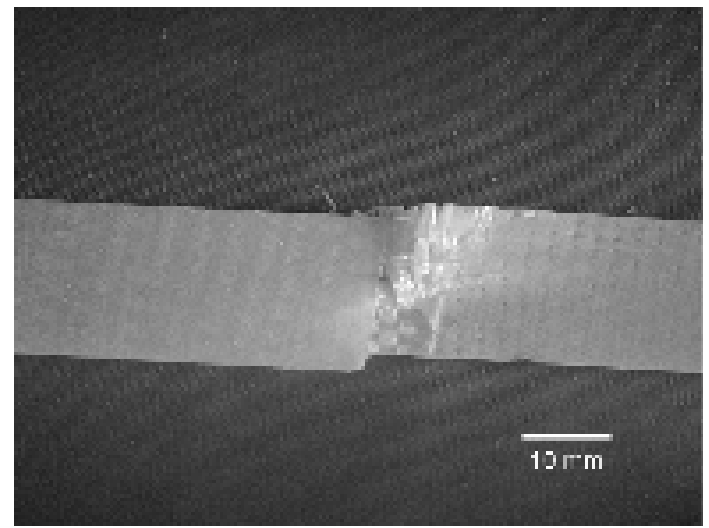

(b) Cure under compression

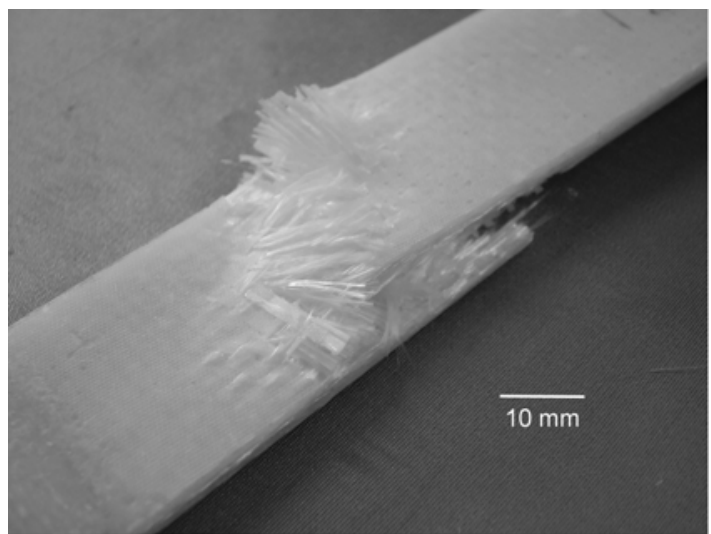

(c) Cure vacuum assisted

Figure 1. Typical failure shape. 
Figures $2 \mathrm{~A}-2 \mathrm{C}$ show dissimilar array formations due to the different cure processes. The black dots are voids formation. Table 1 shows that there are fewer voids when the cure is under compression. The void formation also leads to local rearrangement of tows, which reflects in a non-uniform stress distribution through the thickness. This could guide to premature local failure and therefore affect the composite strength. Moreover, the formation of voids can also affect the fiber volume fraction.

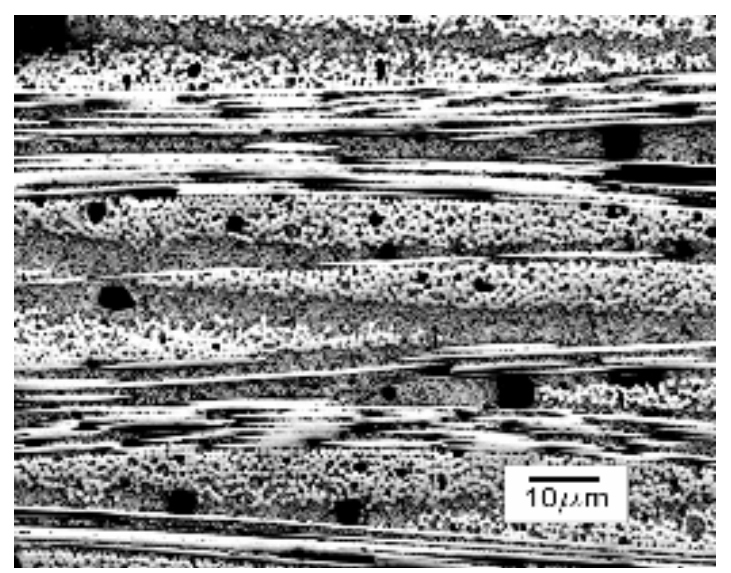

(a) Cure on air

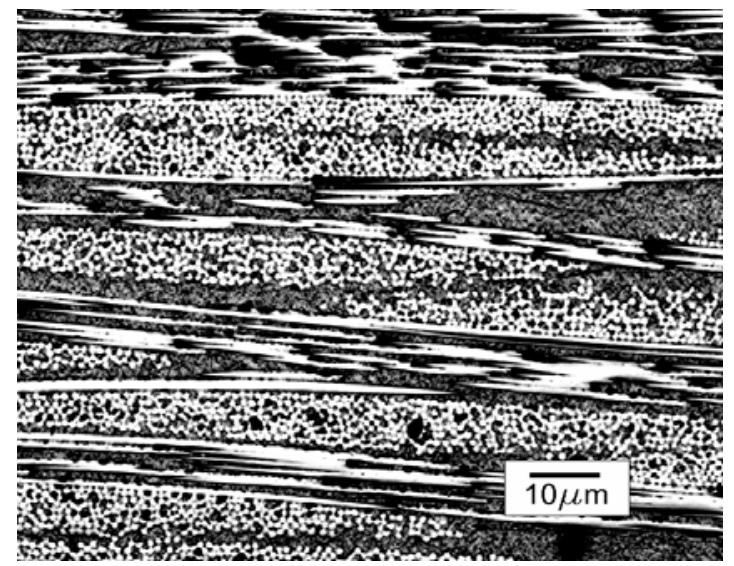

(b) Cure under compression

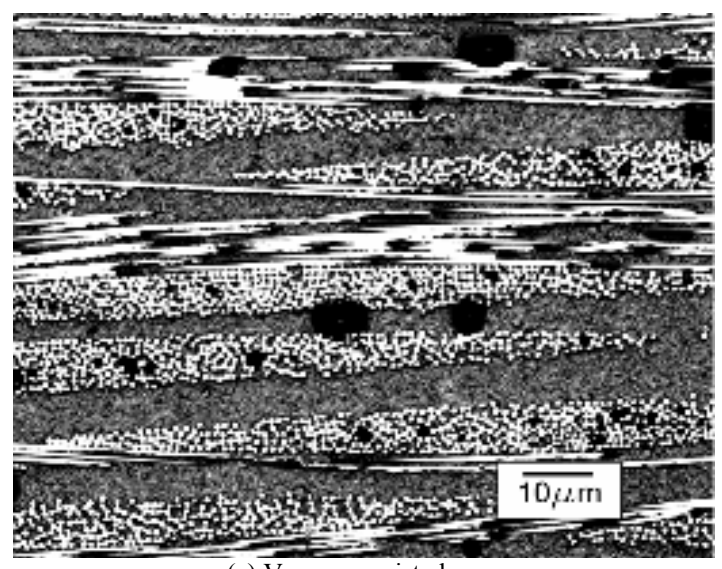

(c) Vacuum assisted cure

Figure 2. Micrographs $(60 \mathrm{X})$ - void formation.

The image analysis shows a slight variation from cure on air to under compression and vacuum assisted, i.e. the volume fractions were $47.86 \%, 49.03 \%$ and $48.15 \%$, respectively. The fiber tow packing factor, however, presents a larger variation. For cure on air, the packing factor is $61.95 \%$, while, for cure under compression, the result is $63.96 \%$ and for vacuum assisted $61.03 \%$. From Figures 3A$3 \mathrm{C}$ it is possible to conclude that the tow packing factor is really a function of the cure process, in other words, the resin infiltration and its location is influenced by the cure process. The large void observed in Figure 3C can be an evidence of a problem during the vacuum formation. This void can be the result of an air bubble trapped during the cure. Note that for the cure under compression, the void sizes are much smaller and homogeneously distributed among the filaments, as it can be seen in Figure 3B. This can be due to the compressive force distribution through the entire composite. The mmTEXlam numerical estimations for all processes studied are listed into Table 2.

Table 1. Void formation versus cure process.

\begin{tabular}{lccc} 
& \multicolumn{3}{c}{ Void [\%] } \\
Mean value & $\mathrm{A}^{\bullet}$ & $\mathrm{C}^{\ominus}$ & $\mathrm{V}^{\square}$ \\
Std. deviation & 2.14 & 0.70 & 1.85 \\
Median & 1.48 & 0.43 & 1.55 \\
Variance & 1.63 & 0.58 & 1.47 \\
CI Mean value & 2.18 & 0.18 & 2.41 \\
CI Std. deviation & $1.45-2.84$ & $0.50-0.90$ & $1.13-2.58$ \\
CI Median & $1.12-2.16$ & $0.33-0.63$ & $1.18-2.27$ \\
(1.17-2.51 & $0.36-0.78$ & $1.08-2.29$
\end{tabular}

$\mathrm{CI}=95 \%$ of confidence interval
$\mathrm{A}^{\bullet}=$ cure on air, $\mathrm{C}^{\ominus}=$ cure under compression, $\mathrm{V}^{\square}=$ cure vacuum assisted

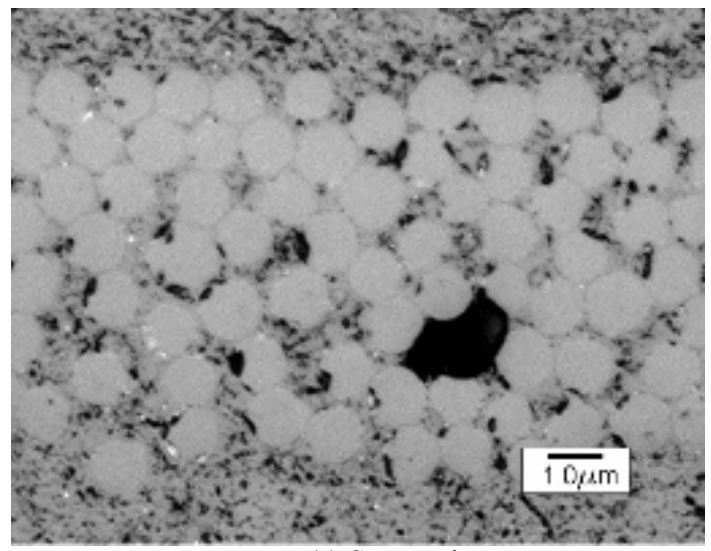

(a) Cure on air

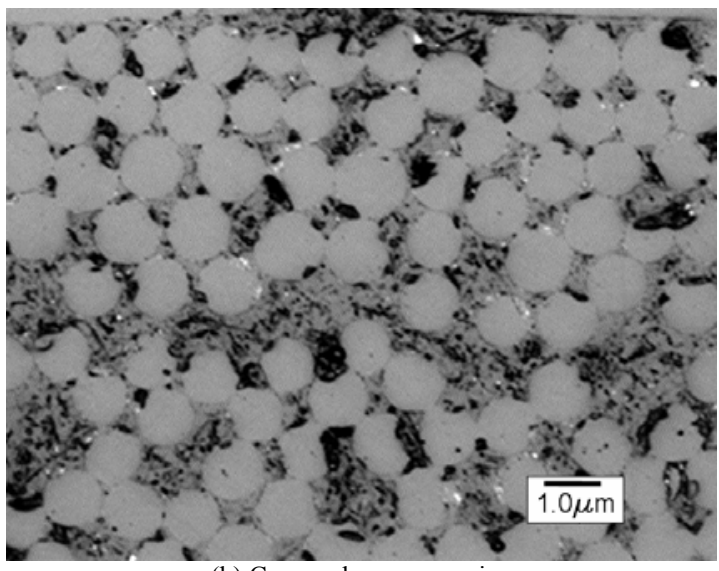

(b) Cure under compression

Figure 3. Micrographs $(800 \mathrm{X})$ - tow packing. 


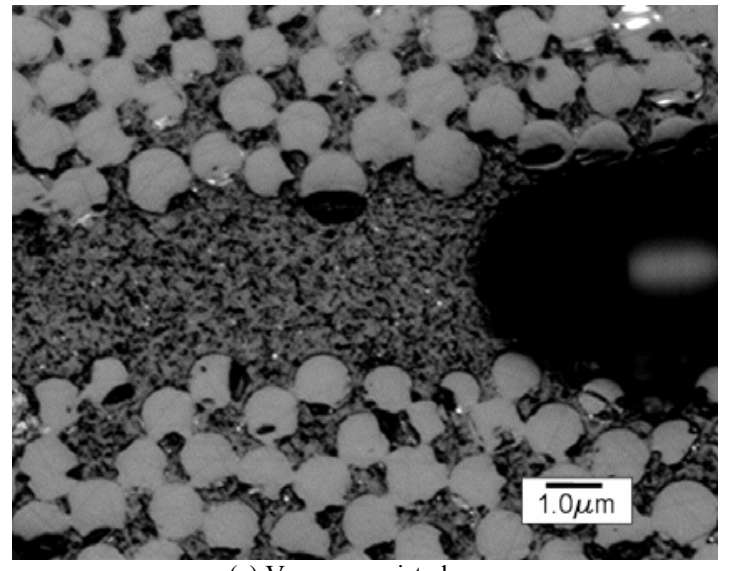

(c) Vacuum assisted cure

Figure 3. (Continued)..

Table 2. mmTEXlam estimates.

\begin{tabular}{cccc} 
Properties & \multicolumn{3}{c}{ Hand lay-up lamination with cure } \\
& $\mathrm{A}^{\bullet}$ & $\mathrm{C}^{\diamond}$ & $\mathrm{V}^{\square}$ \\
$\mathrm{E}_{\mathrm{xx}}[\mathrm{GPa}]$ & 21.980 & 23.060 & 22.380 \\
$\mathrm{E}_{\mathrm{yy}}[\mathrm{GPa}]$ & 21.980 & 23.060 & 22.380 \\
$\mathrm{G}_{\mathrm{xy}}[\mathrm{GPa}]$ & 3.966 & 4.198 & 4.027 \\
$v_{\mathrm{xy}}$ & 0.126 & 0.128 & 0.124
\end{tabular}

$\mathrm{A}^{\bullet}=$ cure on air, $\mathrm{C}^{\ominus}=$ cure under compression, $\mathrm{V}^{\square}=$ cure vacuum assisted

The stiffness results, represented by Young's modulus, are summarized on Table 3. An analysis on Table 3 shows that, although the results are not very dispersed, they don't seem to be equivalent to each other. To be able to check this statement, a series of statistical tests must be performed. The first one is the normality check. The normality charts considering the stiffness are shown in Figures $4 \mathrm{~A}-4 \mathrm{C}$. It seems that none of the three cure processes follows the normal distribution when stiffness parameter is considered. Therefore, as mentioned by Montgomery (2001), Levene's test must be used to check if they have the same variance. Figure 5 is an indication that none of the three cure processes have the same variance, as the $\mathrm{P}$ value is smaller than the level of significance used $5 \%$. As this study deals with three processes with different variances and with a non-normal distribution, the most indicated variance analysis method is Kruskal-Wallis. After performing this non-parametric variance analysis method, the output from MINITAB is shown on Table 4, and it is observed that its $\mathrm{P}$ value is less than the level of significance. Therefore, it is possible to deduce that the three processes must be considered different.

\begin{tabular}{lccc}
\multicolumn{4}{c}{ Table 3. Stiffness versus cure process. } \\
& Young's modulus [GPa] & \\
& $\mathrm{A}^{\bullet}$ & $\mathrm{C}^{\ominus}$ & $\mathrm{V}^{\square}$ \\
Mean value & 17.25 & 19.13 & 17.66 \\
Std. deviation & 0.37 & 0.70 & 0.38 \\
Median & 17.26 & 18.97 & 17.64 \\
Variance & 0.14 & 0.49 & 0.14 \\
CI Mean value & $17.08-17.42$ & $18.81-19.44$ & $17.48-17.85$ \\
CI Std. deviation & $0.28-0.53$ & $0.53-1.01$ & $0.28-0.57$ \\
CI Median & $17.10-17.38$ & $18.70-19.32$ & $17.46-17.72$ \\
& $\mathrm{CI}=95 \%$ of confidence interval \\
$\mathrm{A}^{\bullet}=$ cure on air, $\mathrm{C}^{\ominus}=$ cure under compression, $\mathrm{V}^{\square}=$ cure vacuum assisted
\end{tabular}

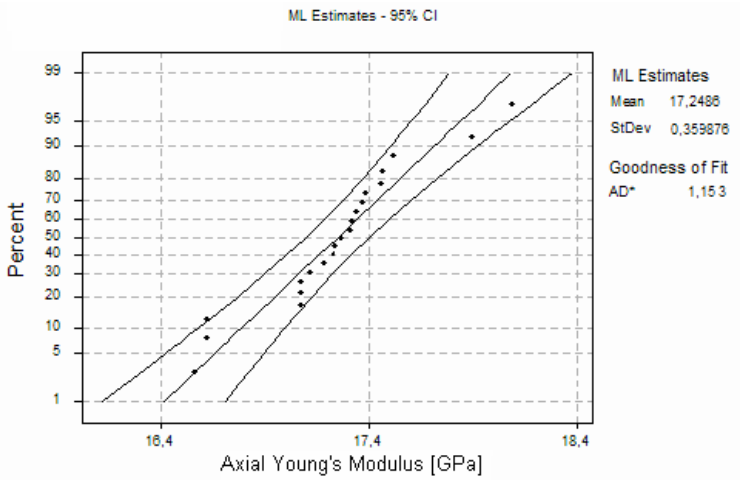

(a) Cure on air

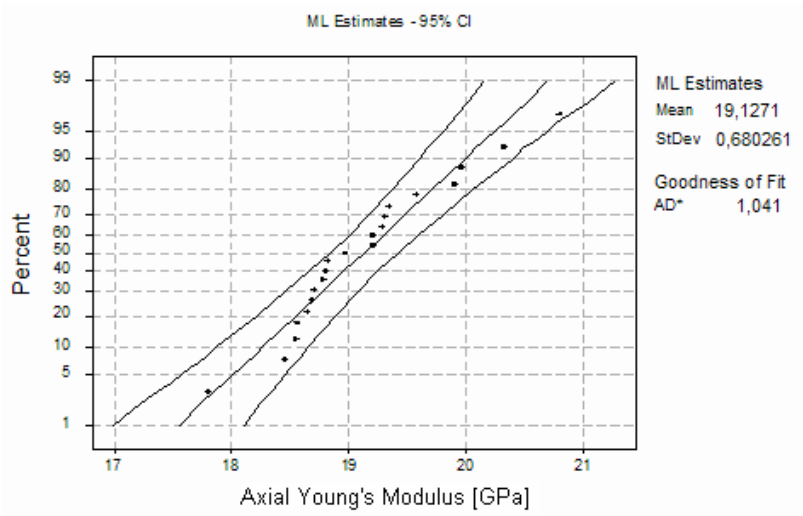

(b) Cure under compression

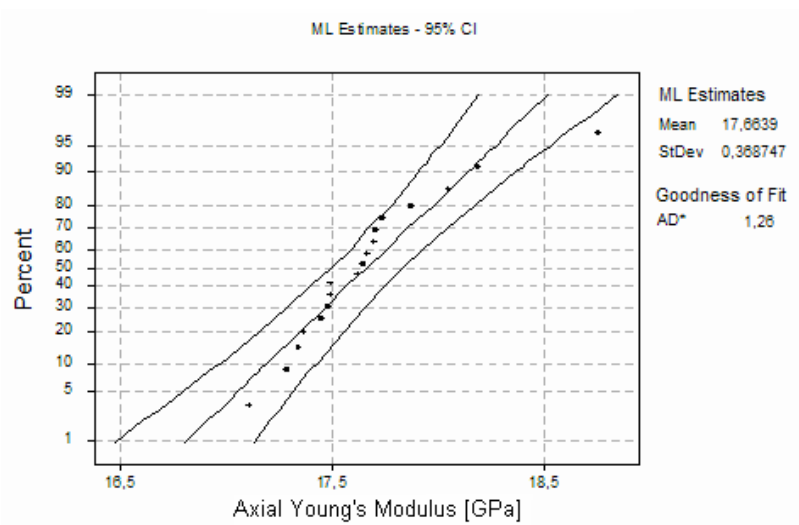

(c) Cure vacuum assisted

Figure 4. Normality charts considering the Young's modulus.

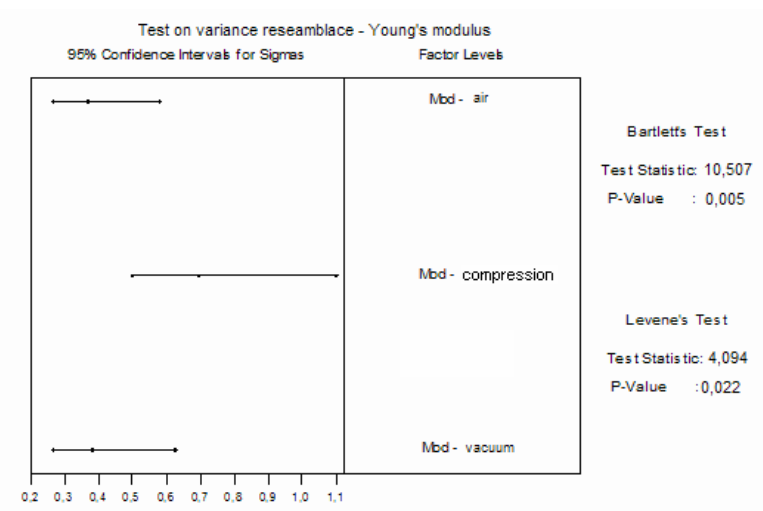

Figure 5. Levene's test for Young's modulus. 
Table 4. Kruskal-Wallis Test on Stiffness.

\begin{tabular}{|c|c|c|c|c|c|}
\hline \multicolumn{2}{|c|}{ Cure process } & $\mathbf{N}$ & Median & Mean Rank & $\mathbf{Z}$ \\
\hline \multicolumn{2}{|c|}{ On air } & 21 & 17,26 & 14,4 & $-5,25$ \\
\hline \multicolumn{2}{|c|}{ Under compression } & 21 & 18,97 & 49,4 & 6,16 \\
\hline \multicolumn{2}{|c|}{ Vacuum assisted } & 18 & 17,63 & 27,2 & $-0,95$ \\
\hline \multicolumn{2}{|c|}{ Overall } & 60 & & 30,5 & \\
\hline $\mathbf{H}$ & 43,19 & DF & 2 & $\mathbf{p}$ & 0,000 \\
\hline $\mathbf{H}$ & 43,20 & DF & 2 & $\mathbf{p}$ & $0,000 *$ \\
\hline
\end{tabular}

As already discussed, the three cure processes will produce composites with statistically different stiffness, even though they have the same volume fraction and the same fiber orientation and stacking sequence. Therefore, the cure process has direct influence on the composite's mechanical properties. Finally, it is desired to know the rate of stiffness reduction as a function of void formation.

Once the micro- and macro-mechanical analysis were performed, it is possible to compute the DYR and the susceptibility coefficient. The composite is made of plain weave woven fabric with the same yarn density in both directions, $\mathrm{x}$ and $\mathrm{y}$, and under plane stress condition. As a consequence, the DYR will be the same for axial and transverse directions. Moreover, as the composite thickness is small, the DYR at $z$ direction can be assumed equal to zero without loss of generality. The susceptibility coefficients $\left(\xi_{\mathrm{i}}\right)$ are listed in Table 5, while Figure 6 shows the $\eta_{\mathrm{i}}-v_{\mathrm{i}}$ for the three cure processes studied. So far, two major conclusions can be draw. First of all, although the percentage voids generated during the cure under compression is reduced to a small percentage $(<1 \%)$, this type of cure is highly susceptible to stiffness variations due to voids. This effect can be translated by a large variation on DYR, and as a consequence on stiffness. Second, a small susceptibility coefficient can be decoded in a small variation on stiffness due to void formation. This is the case for cure on air and vacuum assisted.

Table 5. Susceptibility coefficient.

\section{Cure Process}

On air

Under compression

Vacuum assisted

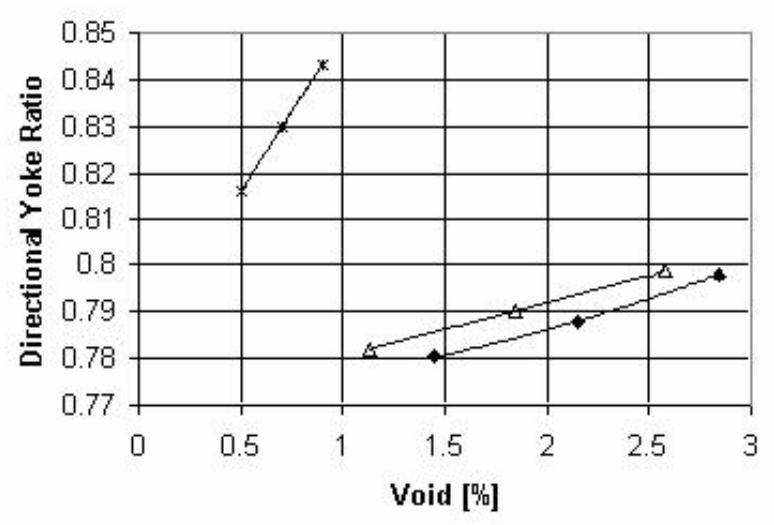

- Air * Compression $\triangle$ Vacuum

Figure 6. DYR versus void formation.

\section{Closing Comments}

A new methodology for composite manufacturing evaluation has been proposed. This new methodology successfully associated the macro-mechanical and the micro-mechanical approaches. Moreover, a statistical study considering variance analysis was also employed during the macro-mechanical tests. By doing this, it is possible to guarantee data consistency.

It was statistically proven that cure on air, cure under compression, and vacuum assisted cure result in different amount of voids and, as consequence, dissimilar stiffness. According to the new methodology proposed, the probability of stiffness variations due to void formation is higher on composites cured under compression. Furthermore, the influence of void formation on stiffness variations of composites cured on air or vacuum assisted is small.

The proposed methodology can be used as a quality control routine for laminated composites. This routine can be easily implemented in an industrial environment. Furthermore, once the concept of continuum monitoring is implemented, the constant retrofitting process can be established.

\section{Acknowledgments}

The authors would like to acknowledge of the financial support provided by Brazilian Research Council (CNPq), the student grant provided by the CAPES foundation and the Graduate Student Program in Mechanical Engineering (PPGMEC) from Universidade Federal de Minas Gerais. The second author would like to acknowledge Professor K. Shivakumar from North Caroline A \& T State University for the use of mmTEXlam.

\section{References}

ASTM D 3039/3039M-00, 2000, "Standard Test Methods for Tensile Properties of Polymeric Matrix Composites", American Society for Testing Materials, Vol. 15, No. 6, pp. 99-109.

Avila A.F, Rodrigues P.C.M, Ribeiro R.P, Oliveira J.H.I, Oliveira M.J.L, 2001, "Plain Weave Woven Fabric Composites: Correlations Between Micromechanical Models and Experiments", In: Proceedings of the $42^{\text {nd }}$ AIAA/ASME/ASCE/AHS/ASC Structures, Structural Dynamics, and Materials Conference, Seattle, WA, CDROM.

Blest D.C, Dutty B.R, McKee S, Zulkifle A.K, 1999, "Curing Simulation of Thermoset Composites, Composites Part A, Vol. 30, No. 10, pp. 1289-1309.

Challa P, Shivikumar K., 2001, “A Graphical User Interfaced Code for Laminated Textile Fabric Composites", In: Proceedings of the $42^{\text {nd }}$ AIAA/ASME/ASCE/AHS/ASC Structures, Structural Dynamics, and Materials Conference, Seattle, WA, CDROM.

Daniel I.M, Abot J.L., 2000, "Fabrication, Testing and Analysis of Composite Sandwich Beams", Composites Science and Technology, Vol. 60, No. 7, pp. 2455-2463.

Fernund G, Rahman N, Courdji R, Bresslauer M, Poursartip A, Wilklen K, Nelson K.,2002, "Experimental and Numerical Study of the Effect of Cure Cycle, Tool Surface, Geometry, and Lay-up on the Dimensional Fidelity of Autoclave-Processed Composite Parts", Composites Part A, Vol. 33, No. 7, pp.341-351.

Golub G.H, Van Loan C.F., 1996, "Matrix Computation”. $3^{\text {rd }}$ edition, John Hopkins UP, Baltimore, 470p.

Gutowski, T.G.P.,1997,“Advanced Composites Manufacturing”, John Wiley \& Sons, New York, 530p.

Han N.L, Suh S.S, Yang J-M, Hahn H.T., 2003, "Resin Film Infusion of Stitched Stiffened Composite Plates", Composites Part A, Vol. 34, No. 2, pp. 227-236.

HUNTSMAN, 2005, "Epoxy Resin Data Sheet", HUNTSMAN Chemical, São Paulo, Vol. 1, pp. 1-14.

Kang M.K, Lee W.I, Hahn H.T.,2000, "Formation of Micro voids During Resin-Transfer Molding Process", Composites Science and Technology, Vol. 60, No. 12-13, pp.2427-2434. 
Kassapoglou C., 1999, "Minimum Cost and Weight Design of Fuselage Frames Part A: Design Constraints and Manufacturing Process Characteristics", Composites Part A, Vol. 30, No. 6, pp. 887-894.

Mallick P.K,1988, "Fiber Reinforced Composites: Materials, Manufacturing, and Design", Marcel Dekker Publishers, New York, 390p.

Mantell S.C, Springer G.S.,1992, "Manufacturing Process Models for Thermoplastic Composites", Journal of Composite Materials, Vol. 26, No. 20, pp. 2348-2377.

Montgomery DC., 2001, "Design and Analysis of Experiments", $5^{\text {th }}$ edition, John Wiley, New York, 490p.

Morais D.T.S, Avila A.F., 2003, "A Study on Laminated Composites Manufacturing Evaluation: The Yoke Ratio Coefficient”. In: Proceedings of the 18th Technical Conference of American Society for Composites. Gainesville, FL, CDROM.
Patel N, Rahatgi V, Lee L.J., 1995, "Micro Scale Flow and Void Formation Mechanism During Impregnation Through a Unidirectional Stitched Fiberglass Mat", Polymer Engineering and Science, Vol. 35, No. 10 , pp. 837-852.

Sawyer L.C, Grubb T.D., 1996, Polymer Microscopy, Chapman \& Hall, New York, 405p.

TEXIGLASS, 2002, "Woven Fabrics Technical Information Sheet". TEXIGLASS Corporation, Sao Paulo,Vol. 1, pp. 1-2.

Um M-K, Daniel I.M., Hwang B-S, 2002, "A Study of Cure Kinetics by the use of Dynamic Differential Scanning Calorimetry", Composites Science and Technology, Vol. 62, No. 1, pp.29-40.

Yoshida H.T, Ogasa T, Hayashi R.,1986, "Statistical Approach to the Relationship between ILSS and Void Content of CFRP", Composites Science and Technology, Vol. 25, No. 1, pp. 3-18. 